\title{
The WHO Surgical Safety Checklist : an Innovative or an Irrelevant Tool ?
}

\author{
D. Vandijck ${ }^{1,2}$, J. Bergs ${ }^{1}$ on behalf of the Flemish Safe Surgery Consortium \\ ${ }^{1}$ Research Group Patient Safety \& Health Economics, Hasselt University ; ${ }^{2}$ Department of Public Health, Ghent \\ University.
}

Innovation in healthcare initiated an exponential growth of treatment options, even for previously incurable diseases. This has led to a longer life expectancy and reduced mortality of patients overall. However, the downside of this progress is the increasing complexity of care, together with a higher risk of complications. Likewise, surgery has become more complex not only due to new innovative surgical techniques, as commonly perceived, but mainly due to the multifaceted non-operative management. Surgery evolved towards a team effort, with multidisciplinary procedures as a key example, which is currently common practice. This evolution in the necessity of team engagement has ; however, opened the spectrum for more (often preventable) adverse events to occur.

With the determination to reduce the number of surgical complications and deaths across the world, the World Health Organization (WHO) launched a Global Patient Safety Challenge entitled 'Safe Surgery Saves Lives' in June 2008. The main goal of this initiative was to strengthen the commitment of operating room (OR) healthcare professionals (respectively, surgeons, anesthesiologists, nurses and other surgical team members) to address safety issues within the perioperative setting. Part of this initiative was the introduction of the surgical safety checklist (SSC), to be applied in any OR and any surgical procedure. The WHO SSC consists of 22 items and identifies three phases of a surgical procedure, each corresponding to a specific period in the normal work flow, i.e. before induction ('sign-in'), before incision ('time-out'), and before the patient leaves the OR ('sign out'). Each phase must be completed by the surgical team before proceeding to the next phase.

The growing evidence has boosted the use of checklists in several areas of healthcare and increased the enthusiasm for a widespread introduction of such tools in clinical practice (1-3). Implementation of the SSC is demonstrated to result in a concomitant reduction in the rate of death and major complications among surgical patients $(4,5)$. Although the totality of evidence is highly suggestive that standardization of care improves patient safety, it cannot be assumed that implementation of the SSC will automatically lead to a reduction in complications. A recent large before-after study showed this concern to be relevant (6). This study concluded that obliged checklist implementation was not followed by a significant effect on postoperative mortality or complication rates (6). Subsequently, a debate whether or not the SSC should be used followed. Is the SSC, herewith, under discussion or not? We believe not. To our opinion, there are several pragmatic issues to consider.

A first and important step to reduce the number of adverse events is to standardize work processes. The probability that crucial steps are omitted or performed at the wrong time during a certain procedure has to be reduced. The easiest way to support healthcare professionals in remembering these crucial steps is to use checklists. Checklists help to identify and correct preventable errors and omissions before things go wrong. Checklists, however, have to be tailored to the local context. On the one hand, they have to be as comprehensive as possible, and on the other hand, they should be short and clear. The WHO allows and promotes modification of the SSC to suit to the local needs of the hospital or to specific discipline related issues, as long as the original items are included. Participation of all involved team members in this process is of key importance to ensure a sense of urgency and ownership. In the light of a new model for hospital inspection, the Flemish government (Belgium) advocated the mandatory use of the WHO SSC, and to be enforced since March 2013. The latter is, of course in good mutual communication, and lasting acceptance among the professionals, appropriate to obtain an overall implementation. Above, implementation of the SSC is also an accreditation standard for hospitals. Here, the need to structurally embed 'quality \& safety' as an issue into the hospital governance DNA strengthens.

Most hospitals currently employ some form of checks in their OR's as part of a safety protocol and there appears to be some general conservative resistance among staff to adapt new innovative systems. Frequently, surgical teams report that the checklist is merely an administrative exercise to meet accreditation standards. Although all Flemish hospitals currently mention to use the SSC, personal communication with surgical teams learns us that while most healthcare professionals are aware of the SSC, it is not being used globally and often performed inadequate. The latter demonstrates that the overall introduction of the SSC involves many challenges, as it requires a change in patient safety culture (7).

Although the general tendency seems quite simple, the vast majority of hospitals are struggling with effective SSC implementation. Are these simply the complaints of a reluctant minority of non-believers or late-adapters, 
and issues to be addressed in an otherwise appropriate adoption of the SSC ? What does the future hold, and how should surgical teams support full compliance with the SSC as a solid confirmation of support for patient safety?

There are several solid reasons suggesting that surgical care is improved following the implementation of a SSC (4). Nevertheless, some essential considerations should be kept in mind. In order to be effective, the checklist needs to be implemented and used according its intentions. In the beginning of the checklist movement, it was suggested that treating this tools as tick-box exercises may lead the field astray. Logically, it is not the act of ticking of boxes that reduces the risk for adverse events, but the performance of the actions it calls for. Checklists contain not only technical elements, but socio-adaptive ones as well. Technical elements such as initiation of antibiotics before incision are discrete, easily implemented actions. Socio-adaptive elements, such as the time-out procedure to discuss critical steps in the surgical plan, involve more than simple actions. They require strong engagement of all team members. As most items of the SSC act as a trigger to engage teamwork and communication, implementation efforts must consequently address patient safety culture. Checklist items like the introduction between team members, to verify the surgical site, and to review critical steps such as assumed blood loss, etc. will achieve no benefit if done merely as tick-box exercises (8). From this perspective, the Ontario study demonstrates how we need not only rigorous evaluations that document the effects of innovative interventions such as the SSC, but also studies that better identify the active underlying mechanisms of effective implementation and true compliance (6). A study from the Netherlands nicely demonstrated the association between completion of the SSC and post-operative death (9). Full checklist completion resulted in statistically significant reductions in mortality compared with partial or non-compliance with the SSC. As such, it is postulated that improvements obtained after implementation of the SSC might have arisen from enhanced teamwork, communication, as well as attitudes related to patient safety culture in the complex entity that is the OR environment $(3,9)$. So, the benefits of the SSC will be realized only if everyone is supportive to the change and if implementation is strict and robust. Sometimes it is argued that the use of a simple checklists might conflict with the perception of a healthcare professionals' own performance. The SSC will, correctly, not improve individual performance. It will not make you a better surgeon, anesthesiologist, or nurse. What it mainly does is improving team performance. The idea to take a one minute break, rethinking previous actions and reflect on some forthcoming steps is not new in healthcare. Most healthcare professionals already perform some kind of reflective actions in their head before conducting an intervention, which is now structured in a checklist.

Often it is argued that the use of the SSC might be superfluous in low-tech and less complex surgical procedures (e.g. cataract surgery) as it is unlikely to see immediate large benefits due to the predominance of low mortality and low morbidity procedures. However, the checklist needs to be seen as more than yet another obliged form or paperwork and viewed instead as a catalyst for change towards a better patient safety culture. Even the ophthalmology OR is a hazard rich environment. Adoption of technology (e.g. SSC) and principles (e.g. crew resource management) to enhance patient safety has also much to offer to these types of (minor risk) surgery. Whilst, for instance, no significant reduction in overall postoperative mortality or complications can be reached, noticeable improvements in the safety processes (e.g. timely use of prophylactic antibiotics) can be obtained after effective introduction of the SSC.

Next, inefficient hospital resources, a lack of expertise in change management, in gathering data, in coaching, and in providing training in teamwork and the appropriate use of the SSC may hamper implementation. Here, learning from colleagues or from best practices might be inspiring. Communication concepts taught in a single lesson or as an isolated message in an attempt to fix an existing compliance problem will be insufficiently retained. The way our current healthcare system is organized demands for new ways to learn and understand. These new ways of educating people require 'innovative' ways of teaching (10). The use of e-learning, simulation, and realistic team-based communication trainings could be valuable in this context and have already shown to effectively improve communication skills in the OR (11).

Lastly, one should keep in mind that effectively improving the safety of our patients requires long-term efforts, and continuous investments, so it is not surprising that promising innovations such as the SSC will only be adopted stepwise.

In conclusion, working according to the standards of care will help surgeons, anesthesiologists, nurses, and other surgical team members to cope with the increasing complexity. The use of a rigorous checklist, such as the $\mathrm{SSC}$, in the rapidly changing OR environment will consolidate all team members aims to enhance both patient safety and clinical professionalism.

\section{Appendix}

Flemish Safe Surgery Consortium : J.Bergs ${ }^{1}$, D. Claeys ${ }^{2}$, I. Cleemput ${ }^{1}$, J.-L.Demeere ${ }^{3,4}$, V.De Troyer ${ }^{5}$, J.Hellings ${ }^{1}$, D. Ramaekers ${ }^{6}$, M. Somville ${ }^{5}$, M. Van Hiel ${ }^{7}$, D. Vandijck ${ }^{1}$

${ }^{1}$ UHasselt, ${ }^{2}$ Collegicum Chirurgicum Belgicum, ${ }^{3}$ Belgische Vereniging voor Anaesthesie, ${ }^{4}$ Verbond der Belgische Specialisten, ${ }^{5}$ ICURO, ${ }^{6}$ Vlaams Indicatoren 
Project voor Professionals en Patiënten, ${ }^{7}$ Vlaamse Vereniging voor Operatieverpleegkundigen.

\section{References}

1. Blot K., Bergs J., Vogelaers D., Blot S., Vandijck D. Prevention of central line-associated bloodstream infections through quality improvement interventions : a systematic review and meta-analysis. Clin Infect Dis, 2014, doi:10.1093/cid/ciu239.

2. Haynes A., Weiser T., Berry W., Lipsitz S., Breizat A., DELLINGER E. et al. A surgical safety checklist to reduce morbidity and mortality in a global population. $N$ Engl J Med, 2009, 360 : 1928-1937.

3. de Vries E., Prins H., Crolla R., den Outer A., van Andel G., van HeLDEN S. et al. Effect of a comprehensive surgical safety system on patient outcomes. N Engl J Med, 2010, 363 : 1928-1937.

4. Bergs J., Hellings J., Cleemput I., Simons P., Zurel Ö., Vertriest S., VANDijck D. Surgical Safety Checklists : an Update. Acta Chir Belg, 2014, 114 : 219-224.

5. Bergs J., Hellings J., Cleemput I., Zurel Ö., De Troyer V., Van Hiel M. et al. Systematic review and meta-analysis of the effect of the World Health Organization surgical safety checklist on postoperative complications. Brit J Surg, 2014, 101 : 150-158.
6. Urbach D., Govindarajan A., Saskin R., Wilton A., Baxter N. Introduction of surgical safety checklists in Ontario, Canada. N Engl J Med, 2014, 370 : 1029-1038.

7. Vats A., Vincent C., Nagpal K., Davies R., Darzi A., Moorthy K. Practical challenges of introducing WHO surgical checklist : UK pilot experience. BMJ, 2010, 340 : b5433.

8. Bosk C., Dixon-Woods M., Goeschel G., Pronovost P. Reality check for checklists. Lancet, 2009, 374 : 444-445.

9. van Klei W., Hoff R., van Aarnhem E., Simmermacher R., REGLI L., KAPPEN T. et al. Effects of the introduction of the WHO 'surgical safety checklist' on in-hospital mortality : a cohort study. Ann Surg, 2012, 255 : 44-49.

10. VAndisck D., Hellings J. Innovation in critical care nursing education. Nurs Crit Care, 2014, 19 : 59-60.

11. Armour F., Bramble J., McQuillan R. Team training can improve operating room performance. Surgery, 2011, 150 : 771-778.

Prof. Dr. D. Vandijck

Hasselt University

Dept. of Patient Safety \& Health Economics

Martelarenlaan 42

3500 Hasselt, Belgium

Tel. : : +3211268714

E-mail : dominique.vandijck@uhasselt.be 